\title{
Drainage des eaux claires parasites par les réseaux sanitaires. De l'observation à l'élaboration d'un modèle prototype
}

\author{
P. Breil (*), C. Joannis, G. Raimbault \\ Division Eau — LCPC Nantes
}

F. Brissaud, M. Desbordes

L.H.M. Montpellier

\section{Contexte et motif de l'étude}

Cette étude traite des réseaux de collecte des eaux usées et principalement des réseaux séparatifs sanitaires. La fonction de ces réseaux est de collecter et d'évacuer, en général vers une station de traitement, les eaux usées, avant rejet dans un milieu récepteur naturel. Mais, par suite d'un vieillissement naturel des ouvrages, d'une mise en œuvre non adaptée, ou d'aléas divers survenant tout au long des dizaines d'années de leur durée de vie, une partie de ces réseaux n'est plus en mesure de rendre les services que l'on attend d'eux. En particulier, ils collectent fréquemment des quantités importantes « d'eaux parasites». Il s'agit en général d'eaux claires, ne nécessitant aucun traitement, mais venant se mélanger aux eaux polluées, et qui saturent et perturbent le fonctionnement des ouvrages de collecte et de traitement [1]. Ces problèmes ont été abordés, au cas par cas, dans le cadre des quelques centaines d'études diagnostic réalisées à ce jour en France [11]. Outre les gros apports ponctuels liés à des accidents ou à des détournements d'usage du réseau, ces études distinguent classiquement deux types d'apport d'eaux claires (fig. 1) plus ou moins diffus :

- les eaux de nappes, en général, qualifiées d'apports pseudo-permanents, en raison de leur persistance dans le temps. Ce sont les infiltrations qui se produisent à la faveur des défauts d'étanchéité dans les parties baignées ou noyées d'un réseau. Elles constituent un apport de base fluctuant au rythme des saisons et relativement peu sensible aux événements pluvieux. Le "débit de nappe »

(*) Actuellement au CEMAGREF, groupement de Lyon. peut être déterminé par différence avec le débit sanitaire, lui-même estimable par différentes méthodes [7], durant les périodes non influencées par les pluies ;

- les eaux de ruissellement, qualifiées d'apports aléatoires, et qui correspondent à des raccordements illicites d'eaux pluviales sur un réseau sanitaire.

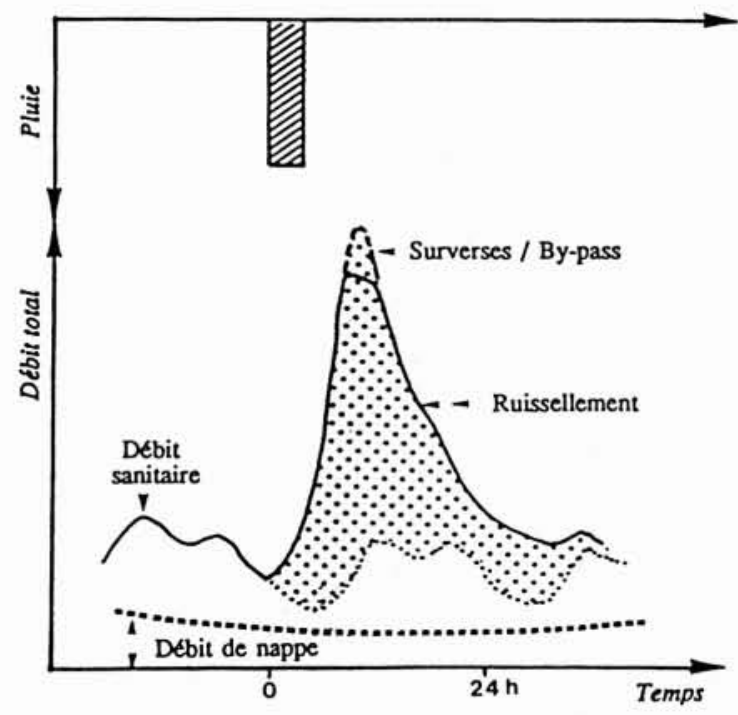

1. Interprétation classique de l'origine des eaux parasites. 


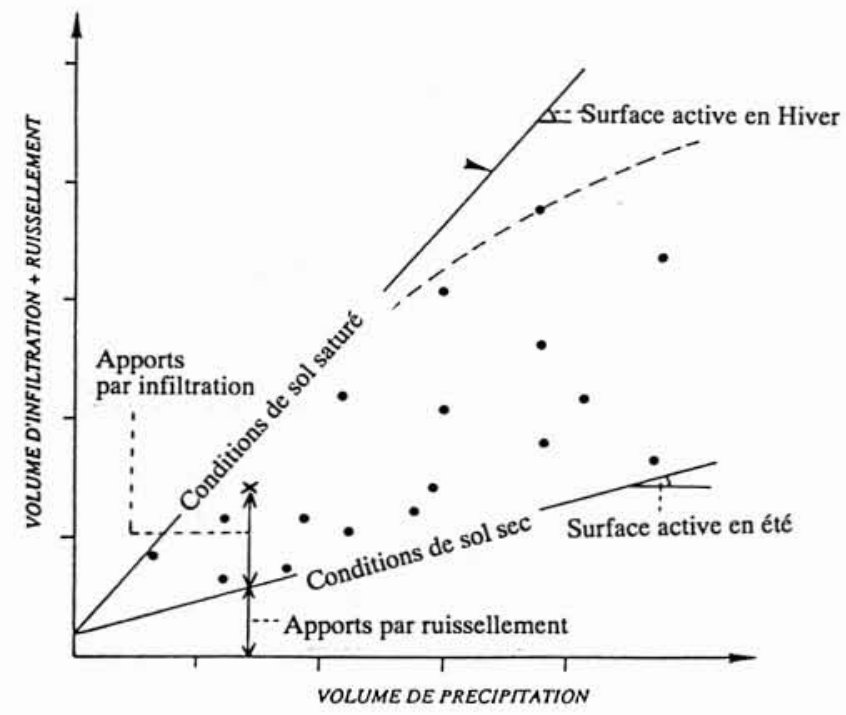

2. Interprétation de la variation de la surface théorique raccordée (East bay I/I study, 1981).

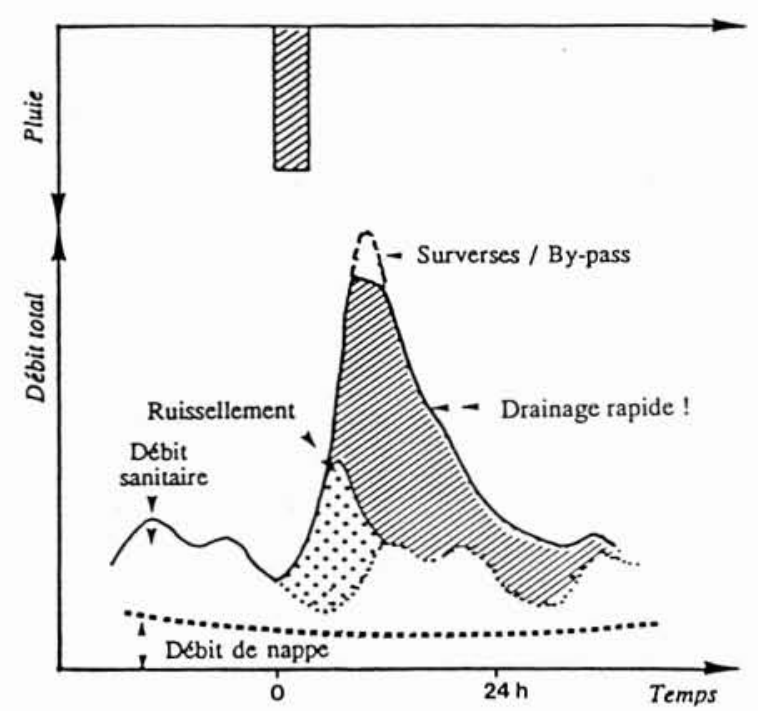

3. Hypothèse du drainage rapide pour une autre interprétation de l'origine des eaux claires parasites.

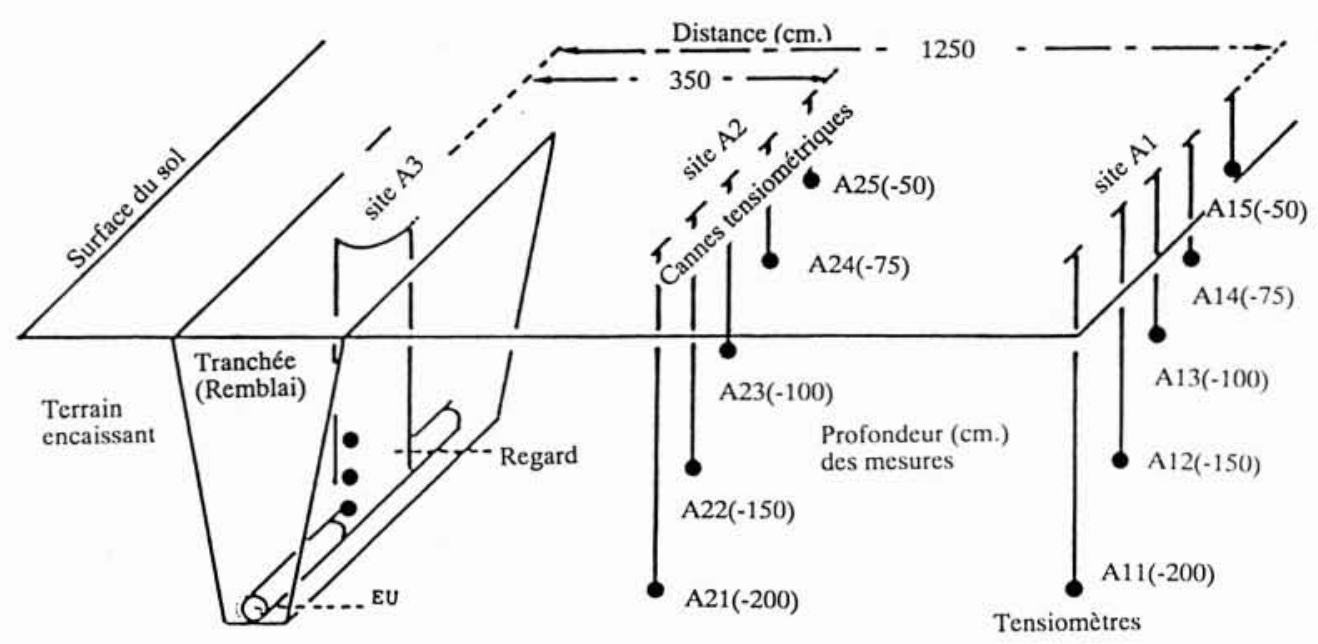

4. Exemple de configuration de suivi tensiométrique du site semi-urbain.

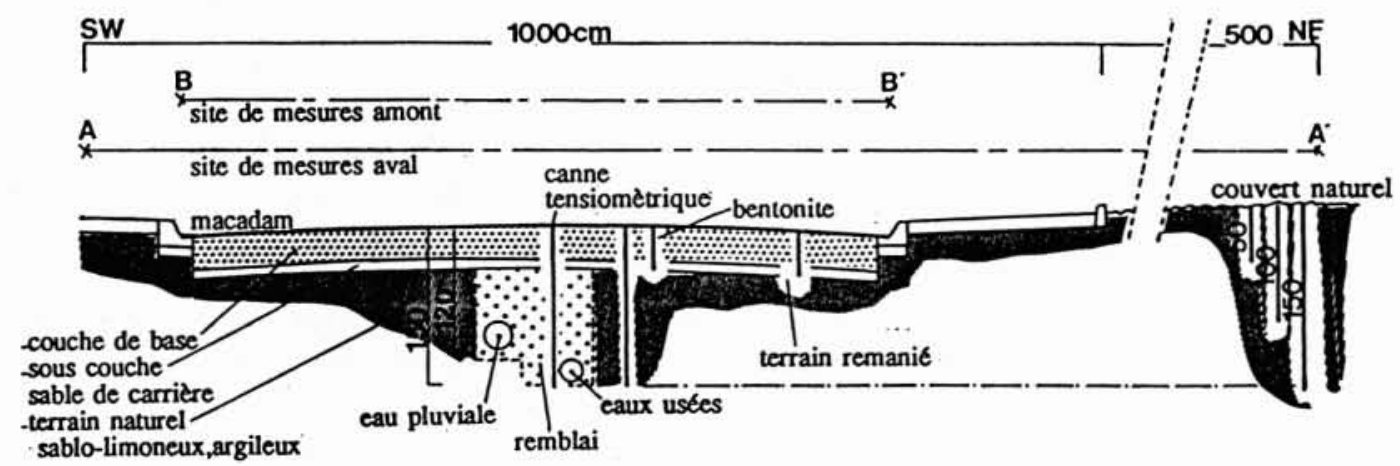

Rue des Bouleaux, lotissement le Praud

5. Configurations des mesures tensiométriques du site urbain. 
Ces deux types d'apport n'ont pas les mêmes conséquences, et ne relèvent pas des mêmes remèdes :

- les eaux de nappes provoquent une dilution chronique des effluents, préjudiciable au traitement biologique [2]. Elles mobilisent inutilement la capacité des ouvrages, surtout à l'échelle journalière. Leur influence est moins sensible sur les débits de pointe mais peut néanmoins poser des problèmes, en particulier au niveau des surverses. Ces apports sont localisés sur le réseau public, et la solution proposée est souvent une étanchéification par injection. Des adaptations du traitement sont également possibles :

- les eaux pluviales provoquent des pointes de débit très importantes, qui s'accompagnent de surverses sur le réseau et de pertes de boues sur les stations biologiques si elles ne sont pas protégées contre ces afflux. Elles sont localisées au niveau des branchements (réseaux privés) et relèvent d'une politique volontariste de mise en conformité, de la construction d'ouvrages de stockage temporaire et éventuellement d'adaptation des ouvrages de traitement.

Il est possible d'observer, dans certains cas, un troisième type d'apports. Très liés à la pluviométrie, comme les eaux pluviales, ils présentent néanmoins une certaine inertie, qui suggère des voies de pénétration comparables à celles des eaux de nappes (joints, fissures, défaut du réseau) ou spécifiques (raccordement de drains). Ces apports sont mentionnés depuis longtemps [1], [12] et qualifiés en général de ressuyage ou inclus dans la dénomination «apports pseudo permanents». En pratique, ils sont rarement pris en compte et qualifiés en tant que tels dans les études diagnostic. Ils peuvent néanmoins apparaître, sous forme de surfaces théoriques raccordées, variables entre été et hiver (fig. 2), ou lorsque les surfaces raccordées sont calculées uniquement sur des mesures hivernales, par un déficit des mauvais branchements effectivement localisés (essais à la fumée) par rapport aux surfaces théoriques. Ainsi, une étude réalisée par le Laboratoire Régional de l'Ouest Parisien [11], sur un échantillon d'une centaine de bassins versants, a montré que le déficit moyen était de l'ordre de $68 \%$. Sur cet échantillon, les branchements directs d'eaux pluviales ne contribuaient donc que pour $1 / 3$ aux volumes d'eaux parasites liés à la pluviométrie. Ce qui est vrai pour les volumes a également pu être observé pour les débits de pointes [3].

Il convenait donc de mieux prendre en compte ce type d'apport que nous avons qualifié de drainage rapide, en proposant une nouvelle interprétation des hydrogrammes liés aux précipitations (fig 3 ), et de trouver des solutions de prévention adaptées.

Certaines des solutions envisageables sont comparables à celles qui sont appliquées pour réduire les apports de nappe (étanchéification par injection), mais elles posent des problèmes pour localiser les zones à traiter, car les apports sont temporaires. D'autres solutions sont plus proches de ce qui est appliqué aux eaux pluviales, car les effets sont comparables (pointes de débit) et la localisation peut être la même (réseaux privés) [3].

Nous avons donc cherché à préciser les mécanismes de ces apports, pour déboucher sur une modélisation ayant les objectifs suivants :
- quantification de ces apports (en débits et en volumes), par extrapolation sur une ou plusieurs années, des mesures effectuées sur quelques mois. Ceci concerne les études de diagnostic, et vise à préciser les conséquences de ce type d'apport sur les surverses, les traitements et les stations d'épuration. Ce type de modèle permettrait de dimensionner des ouvrages de stockage et de surverse en fonction d'objectifs de protection du milieu récepteur bien défini ;

- prévision de ces apports, dans le cadre d'une gestion dynamique d'ouvrages de collecte, de stockage et d'épuration.

\section{Méthodes}

Une observation des phénomènes de drainage rapide avec quantification des débits et des cotes piézométriques a paru indispensable pour progresser.

Deux collecteurs séparatifs eaux usées ont été instrumentés. Le premier, qualifié de «semi-urbain » est situé dans un terrain non revêtu, en bordure de chaussée, mais dont diverses antennes sont situées sous des parkings ou des chaussées. Il a une longueur de $2400 \mathrm{~m}$ (antennes comprises). Le deuxième, situé en zone urbaine pavillonnaire, est qualifié d' "urbain " car implanté sous chaussées. Il a une longueur de $540 \mathrm{~m}$ (hors branchements). Ces deux collecteurs sont en amiante ciment $\varnothing 200$, situés en tête de réseaux, exempts de branchements directs d'eau pluviale. Ils sont situés dans la région nantaise.

\section{Le principe}

L'étude avait pour ambition de relier les débits parasites aux circulations hydrauliques dans le sol. L'expérimentation a consisté à suivre l'évolution du potentiel hydraulique de l'eau dans le sol, à différentes profondeurs et distances des réseaux instrumentés. La pluviométrie et les débits étaient suivis en différents points des réseaux. L'évapotranspiration décadaire était fournie par une station météorologique proche.

Alors que sur le site semi-urbain, les relevés des tensiomètres étaient effectués manuellement tous les 2 ou 3 jours, sur le site urbain on dispose d'enregistrements continus à pas de temps horaire. Sept sites de mesures tensiométriques, comprenant chacun plusieurs profondeurs d'acquisition, ont été installés sur le réseau semiurbain (fig. 4) ainsi que 2 sur le réseau urbain (fig. 5).

Les mesures ont duré quinze mois pour le site semiurbain et 4 mois pour le site urbain, instrumenté tardivement. Pour le site semi-urbain, cela a donné lieu à 8500 mesures tensiométriques et 10500 valeurs de pluie et débit horaire. Ce pas de temps a été choisi car il intègre les temps de réponse et de transfert du réseau semi-urbain et qu'il décrit avec précision les variations de débit. 


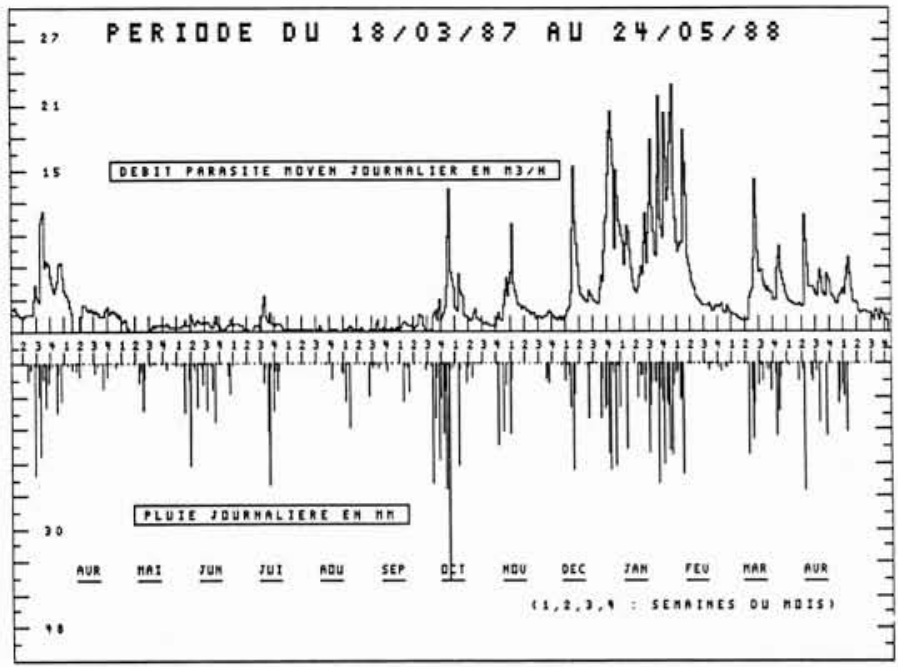

6. Chroniques de la pluie et du débit parasite à l'exutoire du réseau semi-urbain.

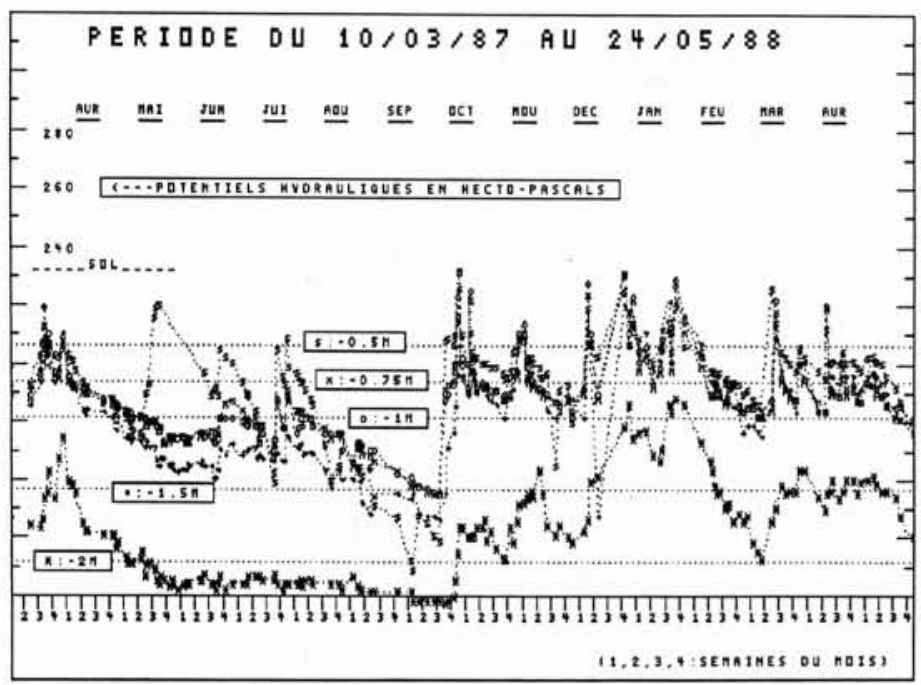

7. Chroniques des potentiels hydrauliques à proximité de la tranchée (Site A2, fig. 4).

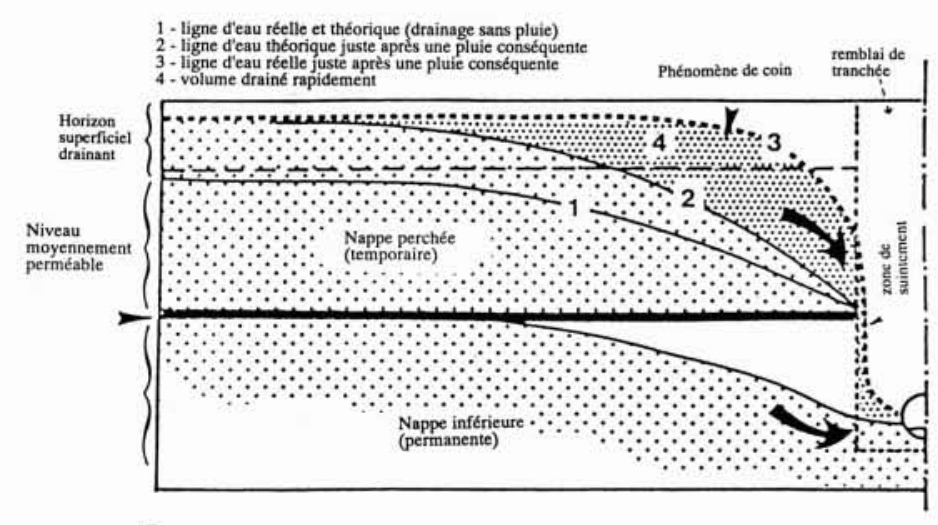

8. Illustration du phénomène de coin en rapport avec le drainage rapide lors d' une remontée de nappe en sol naturel.

\section{Exploitation des résultats obtenus in situ}

\subsection{Le site semi-urbain}

\subsubsection{La relation pluie-débit parasite}

Les chroniques concomitantes des débits parasites et des pluies (fig. 6) mettent en évidence le rôle des saisons sur l'évolution du contexte hydrogéologique des réseaux étudiés.

En l'absence d'apport d'eau pluviale dans les réseaux expérimentaux (apports supprimés quand ils existaient), le calcul du débit parasite de nappe et de drainage rapide s'obtient en soustrayant le débit sanitaire du débit total enregistré.

On constate que le débit parasite augmente en hiver et au début du printemps, par rapport à ce que l'on observe en été pour des pluies comparables.

Une constatation majeure est que l'on observe des pointes de débit malgré l'absence vérifiée de raccordements d'eau pluviale. Le réseau expérimental met donc parfaitement en évidence l'existence du drainage rapide.

\subsubsection{Les potentiels hydrauliques dans le sol au voisinage du réseau}

Le calcul du potentiel hydraulique est référencé au radier du collecteur sanitaire le plus proche. Ce choix donne une lecture directe de la présence de zones saturées susceptibles d'alimenter (localement) le réseau.

La figure 7 est un exemple des résultats des mesures tensiométriques obtenus pendant les quinze mois de suivi. Il s'agit d'un site de mesure situé à $3,5 \mathrm{~m}$ du collecteur sanitaire (Cf. fig. 4). Il est équipé de cinq tensiomètres placés entre 0,5 et $2,0 \mathrm{~m}$ de profondeur. Toutes ces cotes sont rappelées par des lignes pointillées horizontales qui permettent de déterminer si le sol est saturé ou non. Le radier du collecteur est à $2,23 \mathrm{~m}$ de profondeur.

Quelques artefacts de mesure proviennent de succions trop importantes qui provoquent un dégazage de l'eau contenue dans les capillaires. C'est l'explication des fortes valeurs de potentiel hydraulique du mois de mai 1987 pour la mesure située à $0,5 \mathrm{~m}$ de profondeur. De même le gel affecte les quatre mesures superficielles du 22 décembre 1987.

Entre 0,5 et $1,5 \mathrm{~m}$ de profondeur, les potentiels sont peu différents durant pratiquement toute la période d'observation, exception faite de l'inversion estivale qui indique un écoulement ascendant lié à l'évapotranspiration. Le gradient hydraulique vertical tend vers 0 dans l'épaisseur de terrain depuis la surface du sol jusqu'à $1,5 \mathrm{~m}$ de profondeur. Cette tendance est d'autant plus vérifiée que le terrain est saturé. Cette différence de comportement avec le potentiel mesuré à $2 \mathrm{~m}$ de profondeur, caractérise la présence d'une nappe qualifiée de perchée à certaines époques (quand le terrain profond n'est pas saturé), et dans tous les cas d'une nappe supérieure bien individualisée par rapport à celle qui peut exister en profondeur.

L'examen des débits parasites (Cf. fig. 6) montre que les «pointe de débit » sont synchrones des «pointes de 
potentiel » de la nappe supérieure. Si l'on fait abstraction des pointes de débit, le débit de base semble lié au potentiel profond. Ainsi les premières pluies de la fin du mois de septembre (sept.) ne génèrent que des pointes de débit parasite. Il en va de même à la fin du printemps et au début de l'été, dès que le potentiel profond diminue.

On peut critiquer l'extrapolation de mesures locales de potentiel à tout un réseau mais ce type de comportement a été observé pour d'autres sites de mesures tensiométriques sur le même réseau expérimental.

La synthèse de ces observations permet de décrire l'évolution du contexte hydrogéologique à proximité du collecteur sanitaire et fournit une explication au drainage rapide.

\subsubsection{Synthèse des résultats des mesures de potentiel hydraulique}

Une coupe schématique du système collecteur-tranchéeterrain est représentée sur la figure 8.

Les nappes supérieures ou perchées sont dues à des contrastes de conductivité hydraulique verticale dans le sol, qui peuvent être d'origine géologique, pédologique ou artificielle, comme cela a été contrôlé durant l'expérimentation. Ce contraste, ou niveau peu perméable, symbolisé par un trait noir, peut être recoupé par la tranchée d'assainissement. Il correspond sur le site expérimental à des argiles d'altération de la roche mère dans laquelle est située la nappe inférieure.

Les gradients de potentiel horizontaux ont permis d'indiquer les sens d'écoulement (flèches) des nappes supérieure et inférieure (pointillés). Dans le cas de figure présenté, les deux nappes sont drainées par la tranchée d'assainissement, dont le fond se sature et baigne le collecteur sanitaire, d'où les infiltrations d'eau parasites.

En fait, au début de l'automne, le circuit hydraulique peut être différent : la nappe supérieure peut alimenter la nappe inférieure via le remblai de la tranchée d'assainissement, ce qui explique la faiblesse du débit parasite de base alors constaté. La nappe inférieure est donc réalimentée par la tranchée et par drainance, au travers de l'horizon peu perméable, durant une période de transition où l'on observe essentiellement des pointes de débit parasite temporaires.

Ces pointes peuvent s'expliquer par une augmentation rapide mais pas forcément très importante du potentiel hydraulique, en milieu saturé, à proximité du collecteur d'assainissement. Cela implique un drainage rapide des nappes. Un mécanisme à même de l'expliquer a donc été recherché.

\section{Mécanisme du drainage rapide}

On s'appuie pour cela sur l'hypothèse de Boussinesq qui revient à considérer que durant le drainage, la surface piézométrique se déplace par affinité d'axe horizontal passant par les drains et de direction verticale. Le remblai de la tranchée, plus perméable que le terrain encaissant, est assimilable à un drain. On peut alors admettre que le drainage des nappes s'effectue selon des plans parallèles, verticaux, orientés perpendiculairement à la tranchée. La forme de la nappe est donc étudiée dans l'un de ces plans. Les dispositifs tensiométriques situés à une même cote mais à différentes distances de la tranchée, permettent de

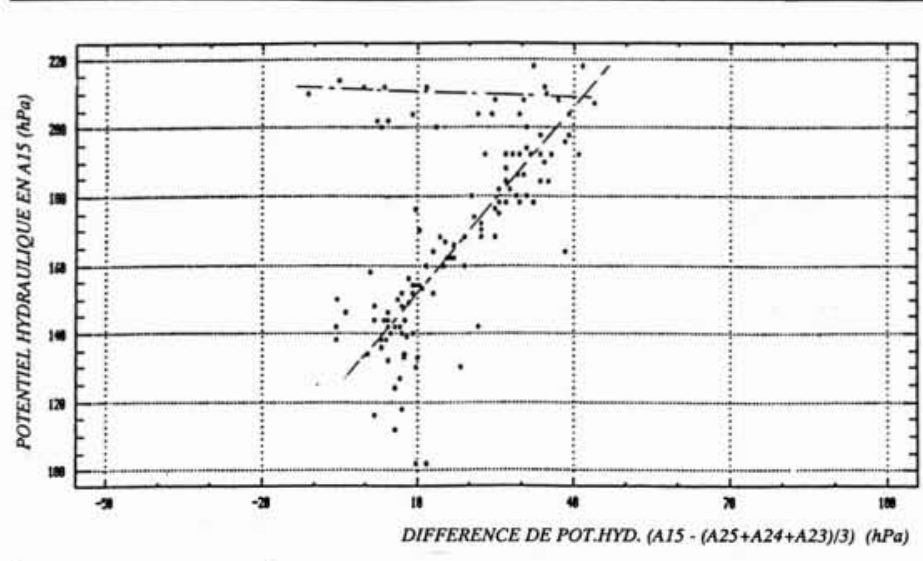

9. Evolution de la "forme de la nappe " au cours du drainage par le remblai de tranchée.

vérifier si l'hypothèse d'écoulement de Boussinesq est applicable à notre problème.

Cette hypothèse est formulée de la façon suivante :

$$
h_{(x, t)}=H_{(t)} F_{(x)}
$$

avec

$t \quad$ le temps

$x \quad$ la distance à partir de la limite d'influence du drainage par la tranchée (rabattement insensible)

$h_{(x, t)}$ la hauteur de la nappe sensu-stricto au-dessus de «l'imperméable »

$H_{(t)}$ la hauteur de la nappe au-delà de la limite d'influence

$F_{(x)} \quad$ fonction décrivant la forme de la nappe.

En dérivant l'équation par rapport à $x$, on obtient :

$$
\frac{\mathrm{d} h_{(x, t)}}{\mathrm{d} x}=H_{(t)} \frac{\mathrm{d} F_{(x)}}{\mathrm{d} x} .
$$

Ce qui signifie que le gradient hydraulique horizontal moyen, mesuré entre deux points situés à des distances $x_{1}$ et $x_{2}$ de la limite d'influence et compris dans le domaine d'influence, est proportionnel à la hauteur de la nappe mesurée à une distance $x$ donnée.

On a vérifié cette hypothèse pour le site présenté sur la figure 4. Le gradient hydraulique moyen a été calculé entre la mesure située à $0,5 \mathrm{~m}$ de profondeur, à $12,5 \mathrm{~m}$ de la tranchée, et la valeur moyenne des 3 mesures situées ente 0,5 et $1 \mathrm{~m}$ de profondeur, à $3,5 \mathrm{~m}$ de la tranchée. La moyenne des 3 mesures est plus représentative de la surface libre de la nappe car il existe un faible gradient vertical entre ces mesures qui résulte du drainage par la tranchée située à proximité.

La figure 9 illustre l'évolution du potentiel hydraulique moyen mesuré à $3,5 \mathrm{~m}$ de la tranchée en fonction de sa différence avec celui mesuré à $12,5 \mathrm{~m}$. La pente approximative du substratum est d'environ $4 \%$ et ne devrait pas trop influencer la forme de l'écoulement. Les mesures de la période estivale ont été éliminées pour clarifier les graphiques. On constate que les mesures expérimentales s'organisent selon une première droite de pente positive jusqu'à une valeur de $165 \mathrm{hPa}$. Cette évolution est conforme à l'hypothèse de Boussinesq ; elle indique que 
la pente moyenne de la nappe augmente avec son épaisseur. Au-delà de $165 \mathrm{hPa}$, les points s'organisent selon une deuxième droite de pente négative. L'hypothèse n'est plus vérifiée car la surface libre tend à devenir horizontale. Ce phénomène a été cité par différents auteurs pour expliquer les débits de pointe en drainage agricole. Il est qualifié de "phénomène de coin " car il s'agit de fluctuations de la nappe localisées à proximité de la tranchée [4], [5].

Dans l'exemple figuré, la hauteur de la nappe se stabilise à une dizaine de centimètres de la surface du sol. La différence de hauteur peut être négative. Pour les faibles potentiels, il peut s'agir d'erreurs de mesure. La différence maximale de $-10 \mathrm{hPa}$ est constatée quand la nappe perchée est affleurante au niveau du site $A_{2}$. Deux mesures correspondent à cette situation ; ce n'est pas forcément représentatif mais en accord avec la topographie du terrain qui est en pente de $A_{2}$ vers $A_{1}$ avec un dénivelé d'environ $10 \mathrm{~cm}$.

Durant les pointes de débit, il a donc été possible d'observer une déformation de la nappe supérieure. Cette déformation correspond, sur la figure 8 , à l'écart existant entre la ligne d'eau théorique (2) et celle déduite des mesures de potentiel (3). Elle est localisée à proximité de la tranchée et contribue à augmenter le gradient hydraulique dans son voisinage. Il est possible d'expliquer cette déformation en constatant que lorsque la nappe supérieure se rapproche de la surface du sol, elle se trouve dans un horizon caractérisé par une conductivité hydraulique horizontale importante qui autorise un écoulement rapide.

C'est le cas, ici, en sol non travaillé, où l'on peut parler de macroporosité. C'est le cas du labour en terrain agricole.

En zone urbaine, les mesures tensiométriques réalisées indiquent que la couche de base des chaussées peut jouer un rôle similaire (les résultats sont présentés au \$ 3.2).

Mais sur le site semi-urbain, les $2 / 3$ amont du linéaire de réseau sanitaire sont en tranchée commune avec un réseau pluvial. Les fuites de ce dernier pourraient donc expliquer tout ou partie du drainage rapide. En fait, cette hypothèse s'est avérée peu réaliste en raison :

- de la persistance du drainage rapide après les pluies, alors que le réseau pluvial était ressuyé ;

- des valeurs de potentiels mesurées dans la tranchée, à la hauteur des collecteurs, qui indiquaient des charges hydrauliques supérieures à celles régnant dans les collecteurs. Dans ce cas il y avait infiltration des eaux du remblai dans les réseaux et non exfiltration des eaux pluviales.

D'après ces résultats, il apparaît que le drainage rapide peut être lié aux fluctuations, elles-mêmes rapides, de la nappe supérieure. Ces fluctuations se produisent en effet dans la partie supérieure du sol, aux propriétés hydrodynamiques favorables aux écoulements rapides. Le débit parasite de nappe dépend alors aussi bien du rabattement des nappes supérieures (hors fluctuations rapides) que de celui des nappes profondes. Ce résultat est compatible avec les variations de potentiel hydraulique constatées et montre que les deux apports parasites ne sont pas complètement indépendants, d'où la difficulté de leurs caractérisations respectives par des mesures physiques. La séparation, dans une perspective utilitaire, des apports par drainage rapide et de nappe comporte donc une part d'arbitraire. Elle ne correspond pas à une séparation physique des écoulements.

\subsection{Les résultats du site urbain}

Le dispositif instrumental équipant la chaussée est résumé sur la figure 5. Il faut noter que le réseau d'assainissement est antérieur à la mise en place de la chaussée ; ce n'est pas un cas général.

L'existence des eaux claires parasites est bien constatée en zone urbaine, tout comme le phénomène du drainage rapide. L'environnement des réseaux d'assainissement urbains, généralement situés sous chaussée, diffère de celui d'un réseau implanté en sol non revêtu (cas du site semi-urbain étudié). Par conséquent, le mécanisme décrit précédemment pour expliquer le drainage rapide devait être vérifié.

Des travaux réalisés sur les variations de l'état hydrique des corps de chaussée [6] confirment que ces structures, plus ou moins poreuses au niveau de la couche de base, subissent un cycle annuel d'humidité. Le contraste de conductivité hydraulique, souvent rencontré, entre sol support et corps de chaussée, favorise l'accumulation d'eau en base de chaussée, d'où la formation de nappes perchées (sensu stricto). Ce phénomène modifie les caractéristiques mécaniques des structures, en particulier sous l'effet des charges roulantes qui produisent des surpressions et la migration des fluides contenus.

L'eau peut s'infiltrer rapidement dans une chaussée à la faveur des défauts de surface qui permettent le franchissement de l'enrobé bitumineux, relativement imperméable (on estime en moyenne la conductivité hydraulique verticale de la couche de surface à $10 E-7 \mathrm{~m} / \mathrm{s}$ ). L'apport par les côtés des structures, à partir des fuites des caniveaux est aussi possible.

Les résultats des mesures effectuées sur le site urbain montrent que le circuit hydraulique entre la tranchée d'assainissement et le terrain encaissant est identique à celui décrit pour le site semi-urbain. En particulier, on retrouve dans la couche de base et au contact du sol support, des nappes perchées qui s'écoulent en direction du remblai de la tranchée d'assainissement.

Cependant, compte tenu de la porosité du matériau (qui peut atteindre $30 \%$ ), les fluctuations piézométriques sont peu perceptibles. Le phénomène de coin n'est a priori pas invocable dans ce contexte mais une transmissivité importante peut jouer un rôle identique.

Une forme d'apport latéraux correspond aux espaces verts qui bordent la chaussée expérimentale. Les gradients de potentiel hydraulique indiquent en effet qu'une nappe se forme dans l'espace vert du site aval (coupe $A$ de la figure 5) et qu'elle est drainée par la couche de base en direction de la tranchée d'assainissement.

Les résultats obtenus en terme de débits parasites, pendant les 4 mois d'expérimentation sur ce site, indiquent une conjonction entre l'évolution des mécanismes et les augmentations de débit.

Parmi les hypothèses à vérifier dans l'avenir, on notera :

- qu'en milieu urbain des circulations préférentielles des eaux de pluie pourraient se produire par les raccorde- 
ments latéraux aux réseaux. En effet, les tranchées de pose de ces branchements sont très superficielles à proximité des habitations et ne bénéficient d'une étanchéité de surface que dans un contexte très urbanisé. Ce n'est pas le cas, en général, des quartiers résidentiels pourtant classés urbains,

- dans les zones à forte urbanisation, par contre, le réseau pluvial transite des débits élevés. Dans ce type de contexte et en réseau séparatif, il est probable que les fuites du réseau pluvial soient en partie responsables des pointes de débit d'eaux claires dans le réseau eaux usées.

\section{Formalisation des relations pluie - débit parasite - potentiel hydraulique}

L'analyse des mécanismes d'apport a été suivie d'une exploitation quantitative des données du site semi-urbain dans le but d'élaborer un modèle de simulation des apports parasites.

La nature du modèle a été choisie en fonction des impératifs opérationnels des études de diagnostic de réseau. Il fallait en effet un modèle relativement simple, ne nécessitant qu'une courte période de calage. Ce sont deux contraintes importantes compte tenu de la complexité des mécanismes de drainage décrits.

L'objectif du modèle était de séparer le drainage rapide des apports de nappe. A partir des résultats exposés ciaprès, le choix d'un modèle global conceptuel, à deux réservoirs, a semblé le mieux adapté pour simuler les deux types d'apport.

\subsection{Bilan hydraulique}

Comme le montrent les résultats précédents, les débits parasites sont en relation avec les fluctuations piézométriques, à proximité du réseau. Pour quantifier ce type de relation et garder l'intérêt des mesures physiques de terrain, un bilan hydraulique a été utilisé. L'objectif en était d'expliquer l'évolution temporelle des potentiels hydrauliques à partir des différents flux connus qui sont la pluie, le débit parasite à l'exutoire du réseau et l'E.T.P. décadaire. Si certains flux non contrôlables n'ont pas été pris en compte (pertes par infiltration profonde, rétention dans le sol, etc...) c'est que leur rôle est apparu négligeable [7].

Le bilan hydraulique est relatif à l'unité hydrologique constituée par le réseau et le terrain encaissant dont la limite latérale correspond à la zone d'influence du drainage induit par la tranchée. Cette limite est estimable, pour le site expérimental semi-urbain, soit à partir des mesures tensiométriques éloignées du réseau, soit en utilisant les volumes d'eau mobilisés par drainage et les caractéristiques hydrodynamiques du terrain. Les deux méthodes indiquent que la zone d'influence du drainage couvre une dizaine de mètres de part et d'autre du réseau.

En toute rigueur, le bilan devrait porter sur des tronçons de réseau homogène en terme de terrain encaissant. La multiplication des postes de mesure débitmétrique que cela aurait impliqué et la variabilité spatiale naturelle de ce contexte ont fait préférer une mesure unique de débit située à l'exutoire du réseau. De cette façon le bilan hydraulique porte sur l'ensemble du réseau et il convient alors de trouver les potentiels les plus explicatifs des variations de débit observées.

$\mathrm{Si}$ les mesures de potentiel sont très locales, la pluie mesurée sur le site et l'E.T.P. fournie par une station météorologique proche, sont considérées comme représentatives pour l'ensemble du réseau semi-urbain.

Pour tenir compte de l'évolution saisonnière du phénomène et de ce que l'information relative aux potentiels hydrauliques est discrète et à pas de temps variable, une formulation intégrale du bilan hydraulique a été préférée à préférée à une formulation différentielle (Cf. encadré).

(Eq. 1) formulation intégrale

$$
H_{(t)}=H_{(t 0)}+A \int_{t 0}^{t} P \cdot \mathrm{d} t+B \int_{t 0}^{t} E \cdot \mathrm{d} t+C \int_{t 0}^{t} Q \cdot \mathrm{d} t
$$

(Eq. 2) formulation différentielle

$$
\begin{aligned}
& H_{t(i+1)}-H_{t(i)}=A \int_{t(i)}^{t(i+1)} P \cdot \mathrm{d} t+ \\
&+B \int_{t(i)}^{t(i+1)} E \cdot \mathrm{d} t+C \int_{t(i)}^{t(i+1)} Q \cdot \mathrm{d} t
\end{aligned}
$$

(*) Dans (2) $t(i+1)-t(i)$ est variable car il dépend des instants où ont été échantillonnées les valeurs de $H_{(t)}$.

$$
\text { avec }
$$

$$
\begin{aligned}
& H_{(t)} \quad \text { : hauteur d'eau ou potentiel hydraulique à } \\
& \text { l'instant } t(L) \\
& H_{(00)} \quad \text { : hauteur d'eau à l'instant initial. Terme constant } \\
& \text { (L) } \\
& \int P, \int E \\
& \text { : intégrales des variables météorologiques pluie } \\
& \text { et évapotranspiration potentielle (E.T.P.) }(L) \\
& \int Q \\
& A, B \quad \text { : coefficients adimensionnels de la régression }
\end{aligned}
$$

\section{Interprétation physique des coefficients :}

L'inverse du coefficient de la pluie brute $(A)$ est représentatif de la porosité moyenne, efficace, du réservoir

$$
A=\frac{1}{\mu(H)}
$$

avec $\mu_{(H)}$ fonction décrivant la porosité verticale équivalente de l'épaisseur de terrain traversée par la nappe au point de mesure.

Le coefficient de l'évapotranspiration potentielle $(B)$ représente la fraction moyenne utile « $F$ (E.T.P.) " ou évapotranspiration réelle (E.T.R.) au facteur près de la porosité. Notons que l'E.T.R. est en toute rigueur fonction de la profondeur de la surface libre des nappes

$$
B=\frac{F(\text { E.T.P. })}{\mu(H)} .
$$

Le coefficient des débits d'eaux parasites $(C)$ a les dimensions de l'inverse d'une surface $\left(L^{-2}\right)$. Il représente la surface active « $S_{a}$ » du bassin qui participe aux apports 


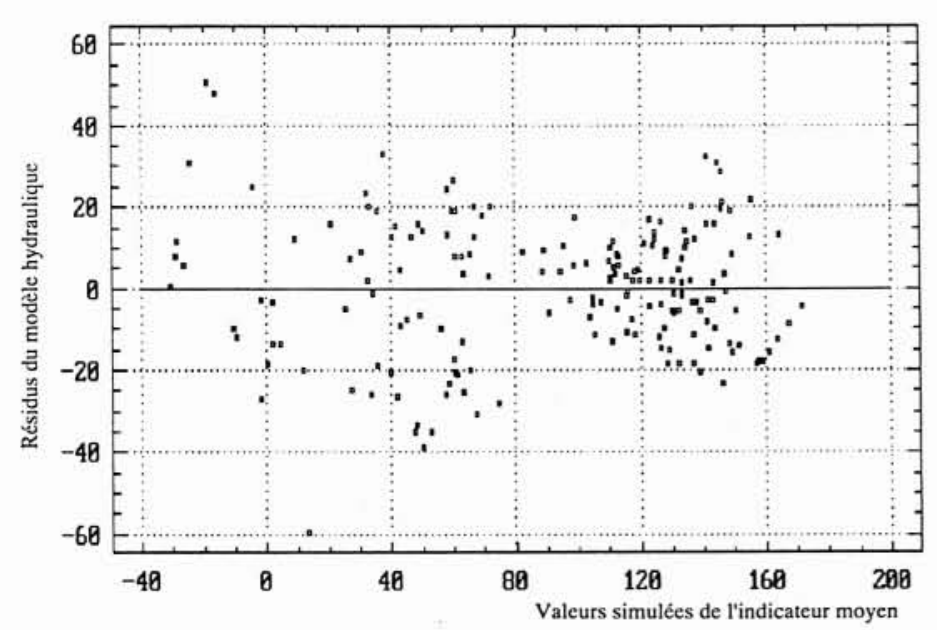

10. Evolution des résidus d'ajustement du modèle de bilan hydraulique en fonction des valeurs simulées.

d'eaux parasites au facteur près de la porosité

$$
C=\frac{1}{S_{a} \mu_{(H)}} .
$$

L'évaluation des coefficients est faite par régression linéaire multiple avec pour variable expliquée, le potentiel hydraulique, et pour variables explicatives, la pluie, l'E.T.P. et le débit d'eau claire parasite.

En raison de la profondeur variable du plancher des nappes temporaires, on observe à partir des mesures de potentiel hydraulique que la «production " des apports parasites évolue progressivement en différents points du réseau au début de l'hiver. Pour tenir compte du décalage temporel ainsi produit et de la discrétisation de l'information spatiale, des indicateurs moyens du niveau des nappes ont été constitués par combinaison linéaire de deux, voire trois potentiels. Le gain de variance expliquée $\left(R^{2}\right)$, par comparaison avec un seul potentiel, est de l'ordre de $10 \%$. On explique ainsi $91 \%$ de la variance d'une combinaison des potentiels représentatifs d'une nappe superficielle et d'une nappe profonde pour la formulation intégrale.

Il faut noter que les intégrales sont de fait autocorrélées. Les coefficients de corrélation partielle ne sont à ce titre pas interprétables pour déterminer si les variables, pluie, E.T.P. ou débit, sont toutes aussi explicatives des fluctuations de l'indicateur moyen. En fait la valeur importante de $R^{2}$ s'explique par la partie saisonnière de l'information qui fluctue lentement au cours du temps. On peut s'abstraire de ces contraintes en travaillant sur la formulation différentielle du bilan hydraulique. Les valeurs obtenues de $R^{2}$ restent toujours moins bonnes, en particulier si l'on conserve la période estivale dans l'échantillon ; les pluies ne générant pas toujours des débits parasites. Le coefficient de corrélation partielle de l'E.T.P. indique dans tous les cas que cette variable n'intervient pas dans les fluctuations rapides de la nappe. Le choix du bilan intégral est donc justifié par le fait qu'il intègre la composante saisonnière des écoulements dont on a vu l'importance dans le cheminement hydraulique des apports parasites.

La figure 10 résume le résultat de la régression multiple sur les différents termes du bilan hydraulique pour expliquer les variations de l'indicateur moyen retenu. Les résidus ne présentent pas de phénomène de dérive particulier et sont donc a priori exploitables. On constate que l'écart entre valeurs réelles et simulées est le plus important pour les valeurs négatives de l'indicateur. C'est logique car les potentiels hydrauliques, qui indiquent dans ce cas un milieu non saturé, sont peu représentatifs des apports possibles des nappes dans le réseau. Pour ce qui est des valeurs maximales de l'indicateur moyen, qui sont intéressantes pour expliquer les pointes de débit parasite, on constate que les résidus augmentent globalement à partir d'une valeur simulée de $120 \mathrm{hPa}$. On ne peut expliquer ce phénomène par la non-concomitance des mesures de potentiels avec les variables explicatives qui sont connues en continu. De même le temps de réponse du réseau et le transfert de l'eau dans le sol sont, d'après les résultats expérimentaux, d'ordre horaire, donc intégrés dans le pas de temps de simulation qui est d'une heure. On peut incriminer dans ce cas la représentativité spatiale de l'indicateur moyen qui pourrait nécessiter une pondération en fonction du linéaire de réseau concerné par les différents types d'apport (nappe perchée, nappe profonde). Cependant cet indicateur améliore les résultats en moyenne et une autre explication peut être avancée : si l'on se réfère au mécanisme identifié du drainage rapide (Cf. \$ 3.1.3), les variations de débit ne sont pas uniquement liées aux fluctuations de la surface piézométrique. Le phénomène de coin a pour conséquence d'augmenter les gradients de potentiel hydraulique et la zone de contact nappe-remblai. La relation débit-potentiel s'en trouve modifiée et il est difficile de l'intégrer dans l'équation de bilan. C'est donc la prise en compte implicite du débit de drainage rapide, terme soustractif de l'équation de bilan, qui est responsable de la sousestimation des maximums de potentiel.

Pour vérifier cette hypothèse, une expression du débit de nappe " $Q_{n}$ " a été recherchée en fonction des potentiels non influencés par les pluies dans les $24 \mathrm{~h}$ précédant les mesures. Les résultats expérimentaux ont permis d'établir que les débits mesurés dans ces conditions sont une fonction sensiblement quadratique de la piézométrie. Cette loi de débit est cohérente avec l'expression classique de la vidange des nappes libres drainées. Sa forme est retenue pour exprimer, dans le modèle, la relation entre le débit de nappe et le stock d'eau, autrement dit la piézométrie.

Cette constatation a orienté la conception du modèle de simulation des débits parasites. Celui-ci comporte deux sous-modèles : un « réservoir nappe " directement lié à la piézométrie et qui simule les débits de nappe, et un "réservoir drainage rapide" (fig. 11). La dénomination de sous-modèle s'explique par le fait que la pluie entre globalement dans chacun de réservoirs. Ce choix confère au modèle de simulation la souplesse nécessaire pour rendre compte de l'évolution du cheminement hydraulique au cours des saisons. En particulier, lors de la reprise des écoulements, le drainage rapide est la source prépondérante d'apport parasite. 


\subsection{Le " réservoir nappe"}

Le « réservoir nappe " est alimenté par la pluie ; il est vidé par l'évapotranspiration et le débit de nappe " $Q_{n}$ ". Le stock d'eau dans le réservoir $\left(S_{n}\right)$, variable représentative de la piézométrie, est calculé par une relation qui a conservé la forme du bilan hydraulique avec pour débit sortant " $Q_{n}$ ". Les équations qui le régissent sont discrétisées au pas de temps horaire.

\section{Calage des coefficients du sous-modèle}

Le calage des coefficients de la relation qui exprime le débit de nappe $\left(Q_{n}\right)$ en fonction du carré du stock $\left(S_{n^{2}}\right)$ est réalisé par régression linéaire sur la période du 18/03/87 au 12/05/87. Cette période est située en début de chronique mais son choix est lié au fait qu'elle est représentative des deux sources d'apport parasite. Cette période contient 27 mesures de potentiel dont 8 correspondent au critère de sélection (absence de pluie dans les $24 \mathrm{~h}$ précédant les potentiels mesurés). Cela peut paraître faible mais la population est bien distribuée en débit comme en stock et permet une première estimation. On obtient alors :

$$
Q_{n(t)}=D \cdot\left(S_{n(t)}\right)^{2}+E .
$$

Le coefficient $E$ peut être différent de zéro et exprimer soit un seuil de stock nécessaire avant écoulement s'il est négatif, soit un apport continu non expliqué par le stock tel qu'on le représente. En général, ce coefficient est faible.

Les coefficients du bilan hydraulique $A, B$ et $C$ (Eq. 1) sont estimés par régression multiple sur la même période avec pour débit sortant " $Q_{n}$ ". Ces coefficients sont

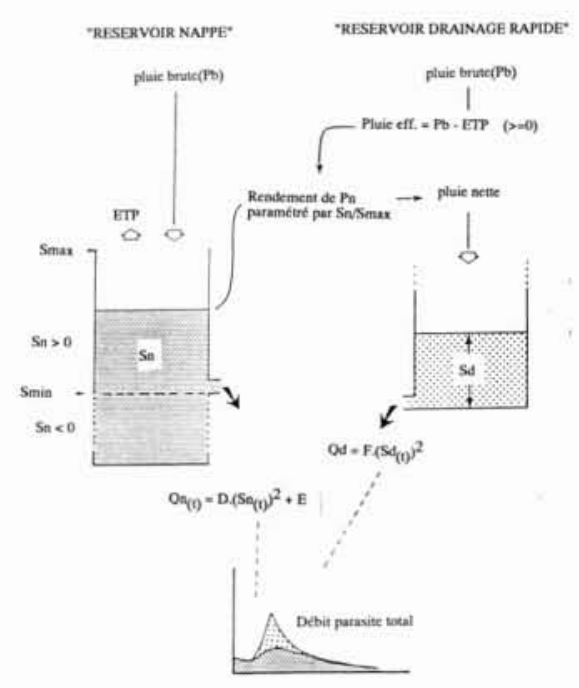

11. Organisation du modèle des apports parasites. retenus comme valeurs initiales dans l'équation (Eq. 4) qui exprime les variations du stock d'eau $\left(S_{n}\right)$ du « réservoir nappe ».

$$
\begin{aligned}
& S_{n(t)}-S_{n(t-1)}= \\
& \quad=A \cdot \text { PLUIE }_{(t)}+B \text {.E.T.P. }(t) \\
&
\end{aligned}
$$

Notons que du point de vue mathématique, les coefficients de la formulation différentielle discrétisée sont strictement identiques à ceux de la formulation intégrale.

A ce stade, la comparaison des débits de nappe simulés et observés sur la période de calage n'est pas satisfaisante en raison vraisemblablement d'une durée arbitrairement choisie de $24 \mathrm{~h}$ sans pluie pour la sélection des potentiels représentatifs des écoulements de nappe. Des essais complémentaires devraient être menés pour définir plus finement cette durée. Cependant la volonté de séparer les apports de nappe et de drainage rapide nous a fait préférer un réajustement des coefficients $A, B$ et $C$ sur le critère de la correspondance des débits de nappe simulés et observés sur la période de calage.

En remarquant que le drainage rapide contribue à augmenter les coefficients de la pluie et des débits et à diminuer celui de l'E.T.P. quelques essais ont été menés. Ils ont permis de constater que seule la modification du coefficient $B$, de l'E.T.P., était sensible et justifiable car sa valeur initiale représentait $12 \%$ de I'E.T.P. et sa valeur finale $67 \%$, ce qui est réaliste. La limite entre débit de nappe et queue de crue étant subjective, un ajustement visuel a été retenu en l'absence de critères formalisables. Le calage du sous-modèle a donc été effectué à la main, par essais successifs, sur la période de calage.

\section{Les paramètres du sous-modèle}

Comme le débit de nappe peut s'annuler en été, le réservoir ne fournit un débit parasite de nappe que pour une valeur minimum, $S_{\min }$, du stock d'eau. Cette valeur est estimée soit à partir des chroniques observées de potentiel-débit, soit pris égal au coefficient $E$ de l'équation 3. Dans le cas étudié, cette valeur était nulle.

Le stock est borné en valeur supérieure $\left(S_{\max }\right)$ pour tenir compte de la réalité de terrain. Cette valeur est estimée en considérant le débit d'apport maximum des nappes au réseau, soit $6 \mathrm{~m}^{3} / \mathrm{h}$ dans le cas du site semiurbain. On pourrait s'en tenir à la «surface du sol » estimée pour l'indicateur moyen mais les chroniques de potentiel-débit indiquent que le drainage rapide apparaît logiquement avant cette limite.

Le calage de ces paramètres $S_{\min }$ et $S_{\max }$ n'a pas été optimisé dans cette première phase de l'étude.

La simulation du débit parasite de nappe permet d'obtenir, par différence avec le débit parasite total, le débit de drainage rapide.

\subsection{Le « réservoir de drainage rapide»}

Le « réservoir de drainage rapide » est alimenté par la pluie nette. Il est vidé par le débit de drainage rapide (fig. 11). 
L'obtention du débit de drainage rapide permet d'analyser les crues. Quinze événements, référencés $\mathrm{A}$ à $\mathrm{O}$, sont ainsi retenus sur la période d'observation. Si l'on reporte les volumes drainés $\left(V_{d}\right)$ en fonction des hauteurs de pluie cumulées $\left(P_{c}\right)$ (fig. 12 ), pour chaque événement, on observe la dispersion des volumes drainés pour une même hauteur de pluie. C'est qu'une partie plus ou moins importante de ces pluies efficaces $\left(P_{e}=\right.$ pluie brute - E.T.P.) est perdue par humidification du sol. Si l'on veut simuler le drainage rapide, il est nécessaire de reconnaître un ou plusieurs facteurs permettant d'exprimer le rendement des pluies.

\section{Expression du rendement des pluies}

En admettant que l'on dispose de plusieurs événements en période de drainage important, on peut définir une " surface active de drainage ». On constate effectivement une tendance maximale des points sur la figure 12 qui permet de tracer une limite supérieure des volumes drainés. Elle correspond au rendement maximum des pluies que l'on relie à des conditions de sol humide. Dans sa première partie elle est linéaire et sa pente donne la surface active « $S_{a}$ ». Pour un événement important « $K$ » le rendement global semble diminuer. Cela peut indiquer une limite aux apports par drainage rapide.

La limite inférieure des événements est attribuée à des conditions de sol sec. Les pertes initiales $\left(P_{i}\right)$, définies comme la quantité de la pluie nette qui ne produit aucun écoulement, sont logiquement plus importantes en condition de sol sec.

L'hypothèse de travail pour exprimer la fonction de rendement consiste à trouver un indicateur qui permette de "ramener" les hauteurs de pluie efficace $\left(P_{e}\right)$ à des valeurs déterminées par le volume drainé $\left(V_{d}\right)$ et la surface active $\left(S_{a}\right)$, c'est-à-dire la pluie nette $\left(P_{n}\right)$. Cette opération est illustrée sur la figure 12, pour l'événement $B$. On essaie d'exprimer la fonction de rendement $R(t)$ représentative des pertes continues de la pluie brute $\left(P_{b}\right)$ en faisant un bilan sur la durée d'un événement

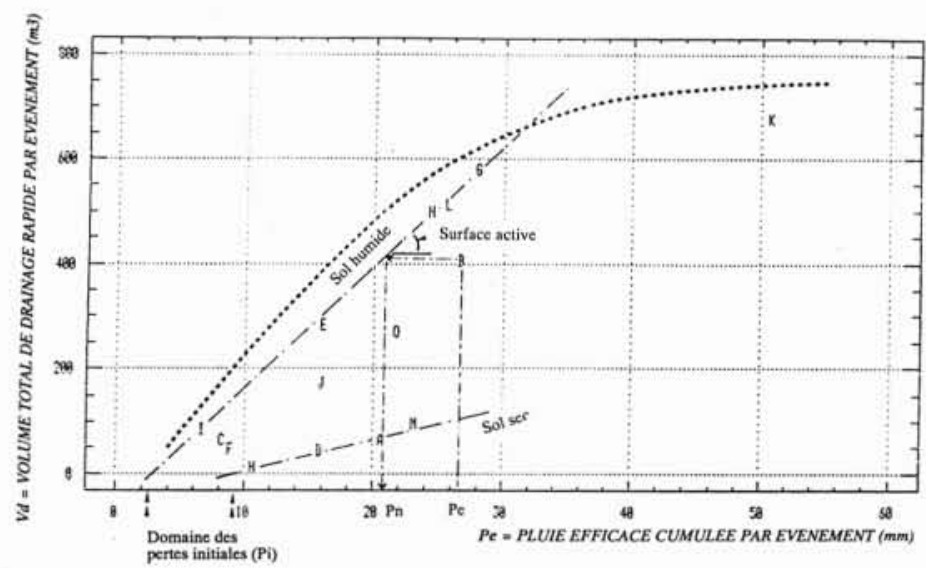

12. Variation du " rendement " des pluies en termes de volume parasite de drainage rapide. crue :

$$
\begin{gathered}
P_{e}=\left(\int \left(P_{b(t)}-\text { E.T.P. }(t)\right.\right. \\
) \cdot \mathrm{d} t)-P_{i} \text { avec } P_{e}>=0 \\
P_{n}=\frac{1}{S_{a}} \cdot \int Q_{\mathrm{d}(t)} \mathrm{d} t=\int\left(P_{e(t)} \cdot\left(R_{(t)}\right)\right) \mathrm{d} t
\end{gathered}
$$

avec intégration entre $t_{0}$ et $t_{f}$, durée de l'événement crue, au pas de temps horaire. Le temps de réponse du réseau étant inférieur à l'heure.

$R_{(t)} \quad$ fonction de rendement des pluies (ou de pertes continues)

$P_{i} \quad$ pertes initiales

$Q_{\mathrm{d}(t)} \quad$ débit de drainage rapide

ETP $_{(t)}$ Evapo Transpiration Potentielle

$P_{b(t)} \quad$ pluie brute

La fonction de rendement $R_{(t)}$ ne peut être simplement déterminée à partir de ces expressions. Puisque l'on veut simuler le drainage rapide avec un modèle à réservoir, on peut exprimer, d'une part, la variation du stock théorique $S$ de la pluie efficace au cours d'un événement crue :

$$
S_{t(t)}=\int\left(P_{e(t)}\right)-\left(\left(1 / S_{a}\right) \cdot \int Q_{\mathrm{d}(t)}\right)
$$

et, d'autre part, la variation du stock $S_{d}$ du réservoir de drainage rapide en introduisant la fonction de rendement $R_{(\ell)}$ de la pluie nette :

$S_{\mathrm{d}(t)}=\int\left(P_{e(t)} \cdot R_{(t)}\right) \mathrm{d} t-\left(\left(1 / S_{a}\right) \cdot \int Q_{\mathrm{d}(t)} \mathrm{d} t\right)$.

Par dérivation par rapport au temps des expressions (7) et $(8)$ on obtient :

$$
\begin{aligned}
\mathrm{d}\left(S_{t(t)}\right) / \mathrm{d} t & =\left(P_{e(t)}\right)-\left(Q_{\mathrm{d}(t)} / S_{a}\right) \\
\mathrm{d}\left(S_{\mathrm{d}(t)}\right) / \mathrm{d} t & =\left(P_{e(t)} \cdot R_{(t)}\right)-\left(Q_{\mathrm{d}(t)} / S_{a}\right)
\end{aligned}
$$

dont on déduit une expression de $R_{(t)}$ :

$$
R_{(t)}=\frac{\Delta\left(S_{\mathrm{d}(t)}\right)-\Delta\left(S_{t(t)}\right)}{P_{\mathrm{e}(t)} \cdot \Delta t}+1
$$

avec $0<=R_{t}<=1$

$\Delta\left(S_{t(t)}\right) / \Delta t$ est facile à obtenir d'après l'équation 7.

Il reste à exprimer $\Delta\left(S_{\mathrm{d}(t)} / \Delta t\right)$. Il suffit pour cela d'établir la relation liant $Q_{d}$ au stock $S_{d}$. On fait l'hypothèse que $R_{(t)}$ tend vers 1 pour un événement crue correspondant à des conditions de saturation importantes du sol.

L’expression du stock étant donnée par :

$$
\begin{aligned}
S_{\mathrm{d}(t)}=\left(\int\left(P_{e(t)} \times R_{(t)}\right) \cdot \mathrm{d} t\right)- & \\
& -\left(P_{i}-\int\left(Q_{\mathrm{d}(t)} / S_{a}\right) \cdot \mathrm{d} t\right)
\end{aligned}
$$

avec

Intégration sur la durée d'une crue $P_{n(t)}$ la pluie nette 
$R_{(t)} \quad$ le rendement de la pluie, ici pris égal à 1

$P_{i} \quad$ les pertes initiales $/ P_{i} \rightarrow 0$ quand $R \rightarrow 1$ (sol humide)

$S_{a} \quad$ la surface de drainage rapide.

La connaissance de $S_{\mathrm{d}(t)}$ permet d'établir la relation liant le débit $Q_{d}$ au stock $S_{d}$. Les coefficients de cette relation sont obtenus par régression $\left(R^{2}=96 \%\right)$. La relation obtenue est de la forme:

$$
Q_{\mathrm{d}(t)}=F \cdot\left(S_{\mathrm{d}(t)}\right)^{2}+\xi \text { avec } \xi \# 0 .
$$

On peut donc rendre compte du drainage rapide par un modèle à un réservoir quadratique :

On peut alors exprimer $\Delta\left(S_{\mathrm{d}(t)}\right)$ en fonction de $\Delta\left(Q_{d}\right)$ :

$$
\Delta Q=2\left(F \cdot S_{\mathrm{d}(t)}\right) \cdot \Delta\left(S_{\mathrm{d}(t)}\right) .
$$

Si l'on dispose de plusieurs valeurs de $R_{(t)}$ il est intéressant d'observer leur corrélation avec un facteur rendant compte de l'état hydrique du sol, comme le niveau des nappes.

L'événement « $B$ » permet de calculer 15 valeurs de $R_{(t)}$ qu'il est possible de corréler linéairement $\left(R^{2}=40 \%\right)$ à un indice hydrique défini comme le rapport de l'indicateur moyen du niveau des nappes à son maximum théorique (rapport compris entre 0 et 1).

$R_{(t)}=G$. (ind. moy./ind. moy. $\max$ ) $+\lambda$ avec $\lambda \# 0$.

Cette corrélation n'a pas été optimisée en utilisant d'autres événements et d'autres indicateurs de l'état hydrique du sol car il s'agissait dans une première étape d'observer la pertinence du modèle proposé dans ses grandes lignes. L'indicateur moyen "représentant » le niveau des nappes, on considère en première approximation que l'indice hydrique peut être décrit par le rapport du stock du « réservoir nappe " $\left(S_{n}\right)$ à son stock maximal $\left(S_{\max }\right)$. Cela permet de paramétrer le rendement des pluies disponibles pour le drainage rapide par le réservoir nappe. La fonction de production qui définit la pluie nette entrant dans le réservoir de drainage rapide s'écrit alors :

$$
P_{n(t)}=P_{e(t)} \cdot\left(G \cdot\left(S_{n(t)} / S_{\max }\right)\right) \text { avec } P_{e(t)}>=0 .
$$

\section{Résultats du modèle de simulation des débits parasites}

\subsection{Adéquation des débits simulés à la réalité}

Le modèle est lancé en période non influencée par la pluie (débit de nappe seulement) et la valeur du stock initial du réservoir nappe correspond à celle de l'indicateur moyen utilisé. La valeur initiale du stock du réservoir de drainage rapide est prise égale à zéro en l'absence de pluie antérieure proche.

La figure 13 présente un exemple de simulation des débits parasites de drainage rapide et de nappe extrait de la simulation réalisée sur les 14 mois d'observation situés hors de la période de calage.
Cet exemple est choisi en raison de l'état de saturation des terrains à cette époque de l'année où l'on peut admettre un fonctionnement homogène des apports parasites au réseau.

Le début de la chronique montre une bonne simulation du débit de nappe. L'apparition du drainage rapide est correctement restituée mais les valeurs simulées sont trop importantes dès lors que le débit de nappe augmente. Ce débit est surestimé du 15 au 19/01/88. On peut critiquer dans ce cas la simplicité de la fonction de production du drainage rapide qui est proportionnelle au taux de remplissage du réservoir nappe. Une fonction non linéaire imposant une évolution moins rapide de la production pour les fortes valeurs de taux (modèle de Gombertz) n'a pas amélioré le comportement global de la simulation.

\section{Amélioration du modèle}

En fait les observations de terrain indiquent que le drainage rapide augmente rapidement avec le niveau des nappes. Il serait donc préférable, d'un point de vue bilan global, de diminuer la part du débit de nappe quand son stock représentatif $\left(S_{n}\right)$ tend vers sa limite supérieure $\left(S_{\max }\right)$. Si l'on ne remet pas en cause le bilan hydraulique, c'est donc au niveau de la pluie entrant dans le réservoir nappe qu'il faut agir. On ne conserverait alors qu'une seule entrée de pluie qui serait répartie en fonction du taux de remplissage du réservoir nappe. A noter que cela ne modifie pas le principe d'évaluation des paramètres du drainage rapide, l'événement de calage étant pris dans des conditions optimales $(R \rightarrow 1)$ où une fraction importante de la pluie participe au drainage rapide. La fraction restante devrait compenser le débit de nappe. Un bilan global des entrées-sorties du modèle serait alors possible.

Un travail de typologie des pluies, en particulier en terme d'intensité-durée, permettrait sans doute de préciser la fonction de rendement des pluies qui est liée à la vitesse d'infiltration dans le sol.

Un moyen de simplifier le modèle est de réduire son nombre de paramètres. Les résultats des simulations ont

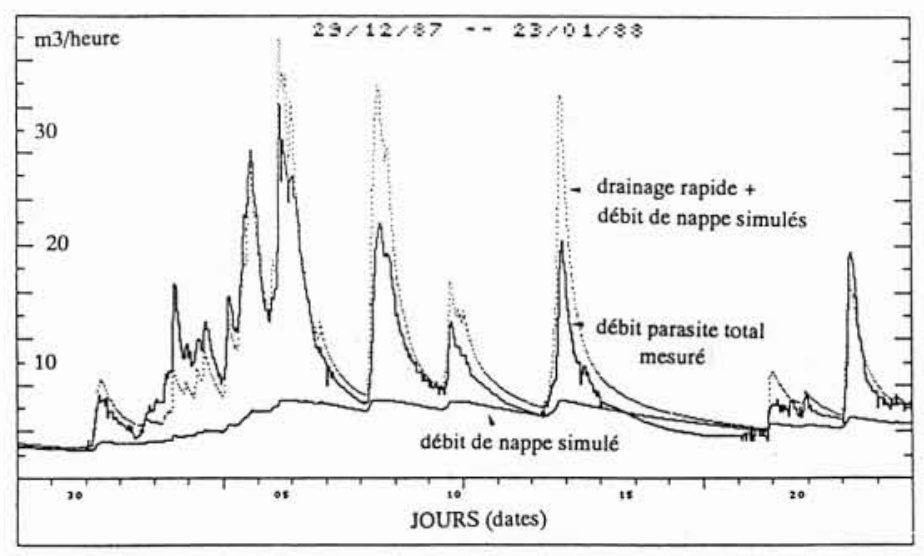

13. Valeurs comparées des potentiels expérimentaux et simulés par le bilan hydraulique. 
montré l'aptitude du schéma conceptuel à reproduire la réalité. Il n'est pas exclu de pousser la réflexion dans ce sens pour définir des relations entre certains paramètres.

Enfin, on s'est surtout attaché à restituer des débits horaires et notamment des débits de pointe. Il faudrait vérifier les performances du modèle dans la restitution des volumes (débits intégrés sur plusieurs heures) car ils constituent une base de dimensionnement pour certains ouvrages, bassin de stockage par exemple.

\subsection{Mise en cuvre du modèle}

On peut résumer les paramètres du modèle comme suit :

\section{Les paramètres du « réservoir nappe»}

$S_{n(t 0)} \quad:$ stock initial

$S_{\max }, S_{\min }$ : stocks $\min$ et $\max$

$A, B, C$ : coefficients du bilan hydraulique

$D, E \quad$ : coefficients de la loi de débit.

\section{Les paramètres du "réservoir de drainage rapide»}

$S_{\mathrm{d}(t 0)}:$ stock initial

$F \quad$ : coefficient de la loi de débit

$S_{a} \quad$ : «surface active » du drainage rapide

$G \quad$ : coefficient déterminant la pluie efficace.

Le modèle comporte donc 12 paramètres en tout. Les 7 coefficients $(A \ldots G)$ sont déterminés par ajustement à partir des données observées. $S_{n(t 0)}, S_{\max }, S_{\min }$ et $S_{a}$ sont déduits des mesures de terrain. $S_{\mathrm{d}(10)}$ est nul si l'on choisit correctement le début de la simulation.
Ce sont en fait les coefficients du bilan hydraulique qui gèrent le réservoir nappe et, par l'intermédiaire du rapport $S_{n}(t) / S_{\max }$, la fonction de production du réservoir de drainage rapide. Les performances du modèle sont donc très sensibles à la détermination de ces trois coefficients.

\subsection{Période de mesure}

Pour le réservoir de drainage rapide, il paraît suffisant de disposer d'une crue observée en condition de nappe haute (hiver). De même pour la fonction de rendement de la pluie, une crue observée en condition intermédiaire (automne ou printemps) semble aussi suffisante. Notons que ce contexte hydraulique est celui de la période de calage du réservoir nappe (printemps) qui requiert des décrues et un étiage.

Pour prendre en compte des eaux de ruissellement présentes dans un réseau sanitaire quelconque, il sera nécessaire de disposer d'événements pluvieux en période estivale. Dans ces conditions, et d'après les mécanismes qui ont pu être observés, on peut espérer modéliser les apports par ruissellement, seuls existant à cette période, à l'aide d'un modèle à réservoir linéaire tel que ceux utilisés en réseau d'eau pluviale [10].

Dans le cadre d'une étude diagnostic, il faudrait donc suivre au moins pendant une année complète, les différents paramètres étudiés, pour un réseau susceptible de subir des aménagements hydrauliques. Selon les performances du modèle on devrait être à même de préciser la durée nécessaire des études diagnostic.

\section{Conclusions}

Sur la mise en évidence des mécanismes de drainage, on peut dire que :

- les sites expérimentaux ont confirmé l'existence du drainage rapide. C'est un résultat important pour les études de diagnostic de réseau,

- les moyens d'investigation (mesures de la pluie, du débit, la connaissance de l'E.T.P.) sont d'accès facile et répondent aux impératifs opérationnels des études diagnostic. La mesure ponctuelle de la charge hydraulique de l'eau dans le sol pose cependant un problème de représentativité. Il n'est pas exclu de s'affranchir de cette donnée par optimisation du calage des paramètres du bilan hydraulique,

- les mécanismes impliqués semblent bien cernés.
Sur le modèle, on peut dire que :

- le réservoir nappe apparaît comme l'élément essentiel du modèle puisqu'il intervient dans le rendement de la pluie qui alimente le réservoir de drainage rapide,

- son utilisation en dimensionnement d'ouvrages de régulation est a priori possible,

- son utilisation pour la gestion des ouvrages de régulation est envisageable, compte tenu de la possibilité de suivre le débit parasite de base (absence de pluie sur plusieurs jours), attribué au réservoir nappe. C'est ce réservoir qui, dans le modèle, conditionne l'importance des maximums de débit observés en hiver,

- développé dans un cadre semi-rural, le modèle doit à terme être applicable aux réseaux urbains. Les premières observations faites sur ces sites sont cohérentes avec celles réalisées en site semi-rural. Il faudra donc tester le modèle sur des sites urbains après l'avoir simplifié. 


\section{Références}

[1] Ranchet J., Renard D., Vicy A. - " Analyse et détection des eaux parasites dans les réseaux d'assainissement ", Techniques et Sciences Municipales (TSM), vol. 77, $\mathrm{n}^{\circ} 4$, avril 1982, pp. 731-183.

[2] Renault D. - « Les eaux claires parasites dans l'assainissement (réseaux unitaires). Diagnostic et réhabilitation des réseaux, impact et économie dans le traitement ", TSM. nov.-déc. 1983, pp. 547-55 et 577-582.

[3] Bishop W., Diemer D., Wallis M. - « Regional infiltration/inflow study solves wet weather sewer problems ", Journal WPCF, vol. 59, n`5, mai 1987, pp. 289-293.

[4] Laurent F., Lesaffre B. - « Etude statistique des débits élevés en drainage agricole ", Etudes du CEMAGREF H.S., $n^{\circ} 6,1983$.

[5] Lesaffre B., Zimmer D. - « Modélisation du comportement hydraulique d'un sol drainé: débits de pointe et tarissement ", Sciences du Sol, vol. 25/4, Plaisir France, 1987, pp. 231-246.

[6] Raimbault G., Silvestre P. — « Analyse des variations d'état hydrique dans les chaussées », Bull. liaison Labo. P. et Ch., 167, mai-juin 1990, Réf. 3504.

[7] BREIL. P. - «Drainage des eaux claires parasites par les réseaux sanitaires. Mécanismes et approche quantitative ", Thèse de Doctorat, L.H.M. Montpellier II, 1990.

[8] Loumagne C. - " Prise en compte d' un indice hydrique du sol dans la modélisation pluie-débit ». Thèse de Doctorat, Uni. Paris-Sud, centre d'Orsay, 1988.

[9] Miouse C. - «Analyse des écoulements sur les petits bassins versants ruraux en vue de l'amélioration de l'assainissement urbain et périurbain ", Thèse de Doctorat, L.H.M. Montpellier II, 1989.

[10] DesBordes M. - "Modélisation en hydrologie urbaine, recherches et applications ", L.H.M. Montpellier II, 1984.

[11] Joannis C. et al. - "Métrologie et études diagnostic », Rapport LCPC - Plan Urbain, juin 1992.

[12] Lavorel. C., Demard H. - « Les eaux parasites dans les égouts sanitaires: diagnostic et réhabilitation», T.S.M. $L^{\prime}$ eau, n' 12, 1979, pp. 615-622.

[13] C.S.T.B. - Centre Scientifique et Technique du Bâtiment, groupe de travail « Réabilitation », données intermédiaires, 1989. 


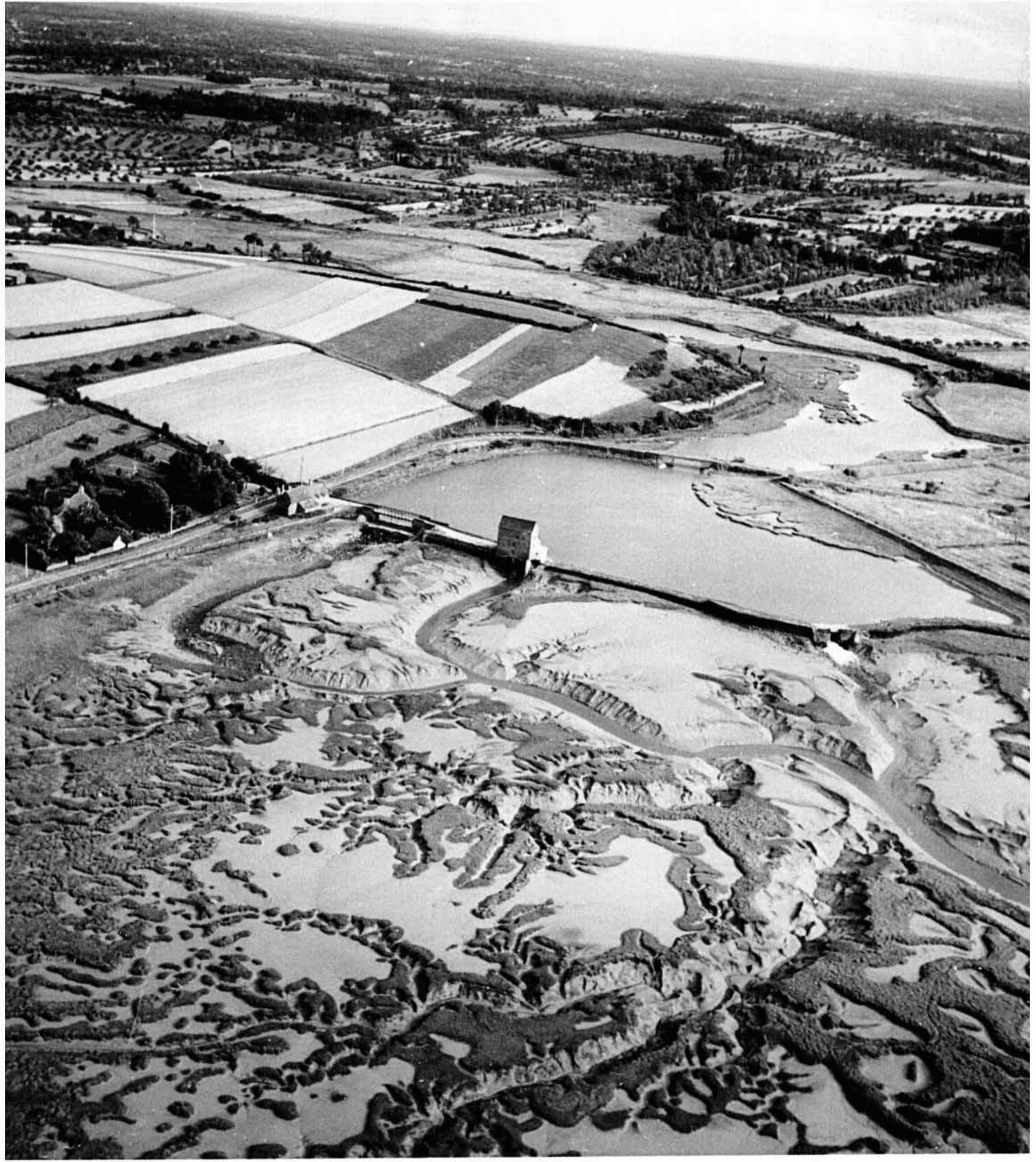

Moulin à marées sur la Rance. 